\title{
The interaction between Greek and Arabic Medical Terms
}

\begin{abstract}
Alia HANAFI
Ain Shams University

Muslims divided science into two distinct categories: First, 'Ilm al$\operatorname{Ady} \boldsymbol{O n}^{1}$ علم الأديان, (i.e. science of religion or of legitimacy): covering

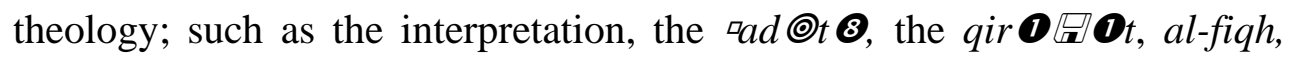
๑elm al-kal $\boldsymbol{O}$, rhetoric, language, syntax, and literature. Second, 'Ilm al$A b d \boldsymbol{O n}^{2}$ علدم الأبدان, i.e. natural sciences or the science of mental or Wisdom. Sometimes, they are called science 'Ilm Al-@A Ojam علم العجم or Ancient science, namely: philosophy, medicine, engineering, astrology, music, magic and chemistry. The second type of science is meant in this paper: namely, the heritage of the Arabs in medicine because medicine is the focus of scientific studies in Islamic civilization, and has a special place and distinct. Arab scholars tries to make science a secular subject and to liberate it from religion.
\end{abstract}

Here I shall draw some of my findings relating to a type of 'Ilm al$\boldsymbol{A b \boldsymbol { d }} \boldsymbol{O} \boldsymbol{n}$ by comparing between the Greek and Arabic medical terms.

Medical terminology has evolved in great measure from the Greek, Latin, and Arabic languages into recent medicine. During the Renaissance period, when the science was begun some medical terms of the disease first used by Galen, and pharmacy by Dioscorides have been retained till now. This accounts for the fact that the second most common source of medical root words is the Greek language. Other older roots have their origins in Arabic. This is due to the fact that Arabic scholars were important teachers of medicine through the middle ages.

The Arabs were the first scientists who had gathered Greek medical 
knowledge's texts into encyclopedias that also contained their interpretations of the texts. Avicenna's al-Canon and Haly Abbas' Pantegn $^{3} i$ are two of the most influential Arab encyclopedias to be translated into Latin. Their works were an ordered catalogue of known medical knowledge.

The new medical texts came with a whole new set of medical terms both from the Greek texts and from its Arab interpreters.

In the Middle Ages, these Arabic encyclopedias were translated into Latin. Since Latin lacked adequate medical terminology, frequently, in the absence of Latin equivalent, Arabic terms were adopted for the nomenclature of the structures of the body in medicine. This gave a historical and scientific value for the Arabic medical heritage.

As it is well known, the medical heritage of the Arab has a historical and scientific value, and it benefits us on the level of the term, and experiences of Arab scientists in this area should be inspirited. The translation of the medical heritage usually distinguish between two different phases: The phase of transference, and readability then the assimilation and understanding.

The phase of transference is prior to the phase of absorption and understanding. Arabs translated medical books from Greek, Syriac, Persian into Arabic, and the phase of absorption began by using the translated knowledge and ended by merging it into the Arabic thought and culture.

The issue of finding the appropriate term in two phases has risen together in varying degrees for finding the accurate Arabic term that will be used in medicine, and is able to express the pure and complex concepts, and to call things by their accurate nomenclature.

In the first phase (i.e. transference and receipt), the main problem for the Arab translators was an almost complete lack of technical Arabic terms in return for Greek terms, and they had to have a solution to the problem by going to three basic ways:

\section{1- The transliteration of the Greek term into Arabic either direct or}




\section{Alia Hanafi}

indirect through the Syriac or Persian language:

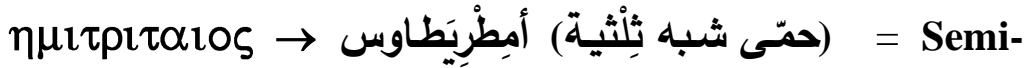
tertian fever

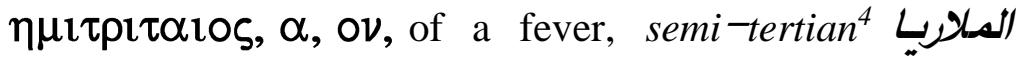

الثلثية:, (see Hippocrates Medicus (v BC.) Epid.1.2, Galenus

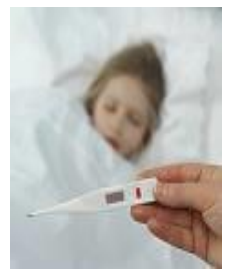

Medicus (ii AD.) 1.17(1) 233), and for $\eta \mu \imath \tau \rho \imath \tau \alpha \iota \kappa o \zeta, ~ \eta, ~ o \nu$, (see Ptolemaeus Mathematicus (ii AD.) Tetr.199).

$\varepsilon \pi 1 \alpha \lambda$ os $\rightarrow$ (حمّى الملاربيا) إِبْيَالوس = Ague

$\varepsilon \pi 1 \alpha \lambda \circ \varsigma$ and $\varepsilon \phi 1 \alpha \lambda \tau \eta \varsigma$, Aeol. $\varepsilon \pi \imath \alpha \lambda \tau \eta \varsigma$ (see Alcaeus Lyricus, iii p. 147 (vii/vi BC.) 129; (= 406 L.-P.), Macrobius Grammaticus (iv/v AD.) Somn.1.3.7, also $\varepsilon \pi 1 \alpha \lambda \circ \varsigma$,

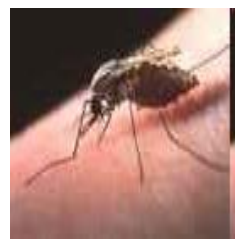

nightmare, conceived as a throttling demon, Phrynichus Comicus (v BC.)1, Dioscorides Medicus (i AD.) 3.140 (pl.). Dor. $\varepsilon \phi 1 \alpha \lambda \tau \alpha \varsigma$ (as nomen proprium), $\mathrm{BCH}=$ suppl. 8, 9, v. index IV. 109.94 no. N7, Artemidorus Daldianus Onirocriticus (ii AD.) 2.37, Rufus Medicus (ii AD.) apud Oribasius Medicus (iv AD.) 7.26.177, Strabo Geographus (i BC. / i AD.) 1.2.8; pr. n. of one of the Aloidae, Il. 5.385, Odyssea 11.308, Salamine de Chypre XIII. Testimonia Salaminia 2: Corpus épigraphique, J. 355 (amphora, vi BC.), Pi. P. 4.89), and Att. pr. n., cf. Aelius Dionysius Grammaticus (ii AD.) Fr.381, Inscriptiones Graecae. $1^{2} .950 .92$, etc. (Identified with $\eta \pi 10 \lambda \eta \varsigma$ by A.D. Fr. 8.12 (or Apollodorus, v. Sophron Comicus (v BC.) 68 note); $\varepsilon \pi 1 \alpha \lambda \tau \eta \varsigma$ (see Suidas Lexicographus (x AD.).

$\kappa \alpha \lambda \circ \pi \circ \delta 10 v \rightarrow$ قالب = caliber

$\kappa \alpha \lambda \circ \pi \circ \delta 10 \nu$ meaning caliber is derived from M. French calibre via the Spanish or Italian language, ultimately, from the Arabic substantive qalib "a mold, last," which perhaps is derived from the Greek substantive $\kappa \alpha \lambda \circ \pi \circ \delta 10 \nu$ "a

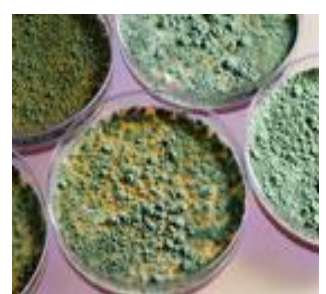


shoemaker's last" lit. It is compound from "little wooden foot" from $\kappa \alpha \lambda \propto \nu^{5}$

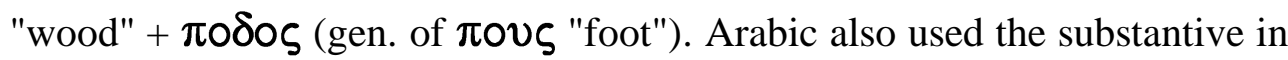
the sense "mold for casting bullets," which is the original lit. meaning in Eng., though the earliest cited sense is the fig. one of "social standing, quality, rank". Calibrate is derived from caliber and is attested as half-life, with means "unsatisfactory way of living;" the sense in physics, "amount of time it takes half a given amount of radioactivity to decay." (see Douglas Harper, Etymology' Dictionary, 2001 vs. caliber. (See Gal. 6.364, Suid. ${ }^{6}$ к $\alpha \lambda \circ \pi \circ \delta \alpha \rho 1 \alpha \mathbf{l} \phi о \rho \mu \alpha \mathbf{l}$ lasts, and Edict.Diocl.9.1. ${ }^{7}$ ).

$\pi 1 \sigma \tau \alpha \kappa 1 \alpha \rightarrow$ pistachio

$\pi 1 \sigma \tau \alpha \kappa \eta$ meaning pistachio الفستق, ultimately from Greek $\pi \imath \sigma \alpha \kappa \eta, \eta$, pistachio tree ${ }^{8}$, Pistacia vera, Alciphro Epistolographos (iv AD.) 1.22 ( See Diosc. I 124 $\pi \imath \sigma \tau \alpha \kappa 1 \alpha$ vs., and Ibn Sina II 412, 5.)

$\sigma \alpha \gamma \alpha \pi \eta v 0 v \rightarrow$ سكَخْبنَاج (Persian) $\rightarrow$ (Arabic) = Ferula persica

$\sigma \alpha \gamma \alpha \pi \eta \nu 0 v, \tau o$, a plant, probably, Ferula persica, Galenus op.cit. 12.117; also its gum, Dioscorides op.cit. 3.95; and as adj., o $\pi \circ \varsigma \sigma \alpha \gamma \alpha \pi \eta \nu \circ \varsigma$ Gal. 1.c. 13.567.

$\alpha \psi \imath v \theta \imath \mathrm{t} \rightarrow$ أفسنتين = wormwood

$\alpha \psi \imath v \theta \imath o v, \tau o$, wormwood, Artemisia Absinthium, Hippocrates Medicus (v BC.) Morb.3.11, Mul.1.74, Xenophon Historicus (v/iv BC.) An.1.5.1, Theophrastus Philosophus (iv/iii BC.) HP1.12.1, Dioscorides (i AD.) 3.23. Another kind called $\alpha \psi \imath v \theta \imath o v \theta \alpha \lambda \alpha \sigma \sigma \imath \mathrm{v}$ (see Dsc.3.23,5).

\section{2- Literal translation of the term:}

$\alpha \lambda \omega \pi \varepsilon \kappa 1 \alpha \rightarrow$ داء الثعلب = (fox sickness or alopecia)

$\alpha \lambda \omega \pi \varepsilon \kappa 1 \alpha, \eta$, disease, like manage in foxes, in which hair falls off (see Sophocles Tragicus (v BC.) Fr.419, and Gal.12.381), pl. bald patches on the head (see Aristoteles Philosophus (iv BC.) Pr.893 38 .
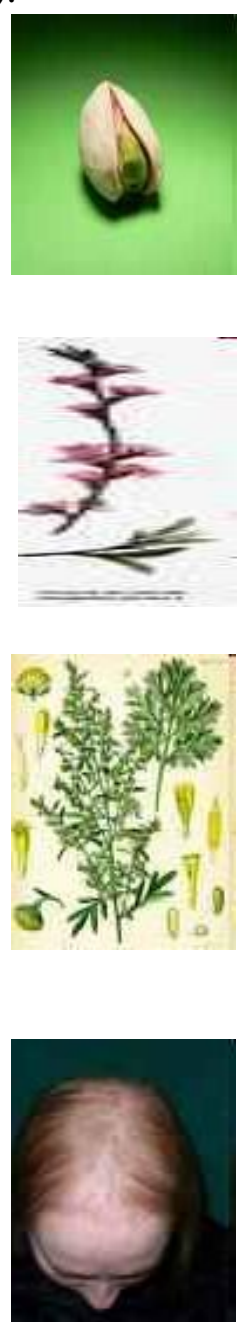

77
79 


\section{Alia Hanafi}

oфı $\alpha \sigma \iota \varsigma \rightarrow$ داء الحية = (snake sickness)

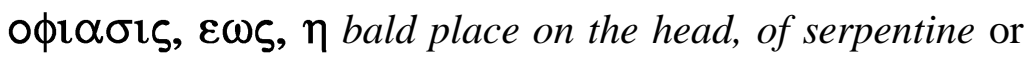
winding form, Gal. 12.381, 10.1004, and a form of leprosy in which the patient sheds his skin like a snake, Ps.Gal.14.757.

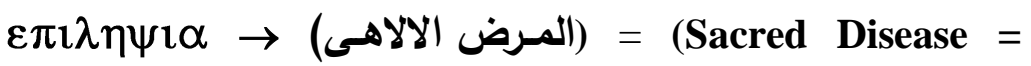
epileptic fit $=$ عرالصر))

$\varepsilon \pi \imath \lambda \eta \psi \imath \alpha, \eta$ or $\varepsilon \pi \imath \lambda \eta \psi \imath \varsigma, \varepsilon \omega \varsigma, \eta$ epileptic fit $^{9}$, (see Hippocrates Medicus, Coac.587, Morb.Sacr.10, Aristoteles Philosophus, Pr.960 18, etc. (but, invasion, attack of disease, Hp.Morb.3.16.).

$\mu v 0 \varsigma \omega \tau \alpha \rightarrow$ أذان الفار = madwort

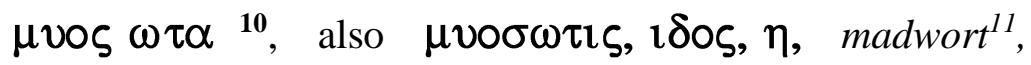
Asperugo procumbens, Dioscorides (i AD.) 2.183. It called also $\alpha \lambda \sigma \mathrm{\imath} \eta, \eta$, lich-wort, Parietaria lusitanica, Theophrastus Philosophus (iv/iii BC.) HP9.13.3, Dioscorides

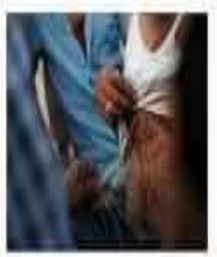
(i AD.) 4.86 .

$\alpha \mu \beta 1 \xi \rightarrow$ الإنبيق = Alembic

$\alpha \mu \beta 1 \xi$ meaning alembic: In modern language, the substantive 'alembic' is the Arabic substantive al-anbiq means 'still' (the distillation device). It is derived

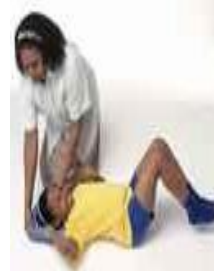
from Greek $\alpha \mu \beta 1 \xi$, meaning a vessel narrowing towards the

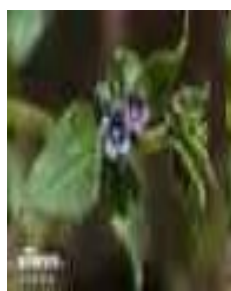
brim, or cup (see Hesychius, Lexicographus (c. v AD.), Athenaeus.11.480d). We have also $\alpha \mu \beta 1 \kappa o \zeta$, cap of still (see Posidonius Historicus (II/I BC.) 67.25 E.-K., and Boeckh A., Corpus Inscriptionum Graecarum, Berlin (1828-77). 3071.7, and Etymologicum Magnum 80.18, as cap of still (see Dioscorides op.cit. 5, 95, Zosimus, Alchemista (iii / iv AD.) p.141 B.).

$\alpha \mu \beta v \xi$, perhaps $=\alpha \mu \beta \imath \xi$, (see Herodianus Grammaticus (ii AD.) 1.44, al).

It is to be noticed that that Greek letter $\mu$ becomes $\dot{\nu}$ in Arabic language 
because when the letter immediately followed by the letter بithout any vowel coming between them, ن takes the sound of as ambar not (i) nbar ${ }^{12}$.

\section{3- Arabicised the Greek terms:}

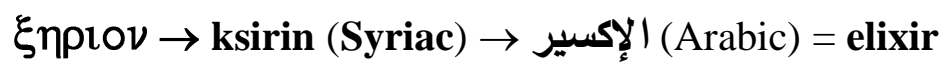

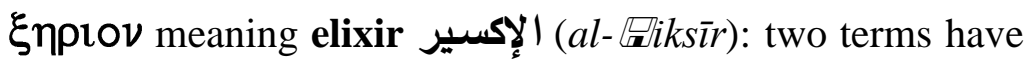
been called elixir: (1) the philosopher stone ${ }^{13}$; (2) medicinal potion (L. medicamentum siccum). The Arabic substantive is derived from Greek $\xi \eta p 10 v$ "desiccative powder for putting on wounds" (see P. Oxy. 1142.7 (iii AD.) ${ }^{14}$ and Alex.

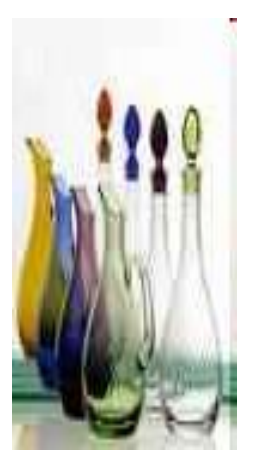

Trall.1.15 $5^{15}$, The substantive can be written without iota (see Aet. 6.65 al., $\xi \eta p o v^{16}$; Alexander Aphrodisiensis Philosophus (iii AD.) Pr. 1.150). The transfer of the Greek medical term into Arabic has two verbal types; either direct from Greek or indirect from Greek through Syriac or Persian.. Thus, the term إكسير is subjected to some Arabic rules, (1) (كسير on the measure of If@ @ (وزن إفعيل), (2) A syllable cannot begin with two consonant, the first of which is destitute of a vowel, as $s f$ or $f l$. Foreign words, which commence with a syllable of this sort, on passing into the Arabic language, take an additional vowel $(\text { alif })^{17}$, usually before the first

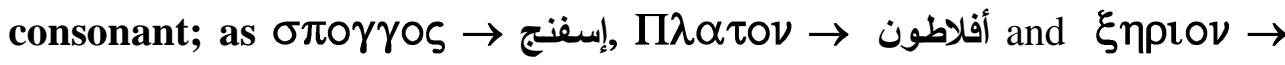
(إكسير Sometimes the final ending of the Greek substantive is elision such as $\xi \eta \rho 10 v \rightarrow$ إكسير or left such as $\alpha \sigma \alpha \rho 0 \nu \rightarrow$ أسارون $\sigma \tau \alpha \xi \rightarrow$ استَّص (استقصات . drop $\sigma \tau \alpha \xi$, perhaps from $\sigma \tau \alpha \gamma \omega \nu$, ovos, $\eta$ drop (irreg. Nom pl. $\sigma \tau \alpha \gamma \varepsilon \varsigma$ ) (see Apollonius Rhodius Epicus (iii BC.) 4.626). It is to be noticed that the Arab scientists used this substantive in its plural form. So استَقصات was arabicised by using the

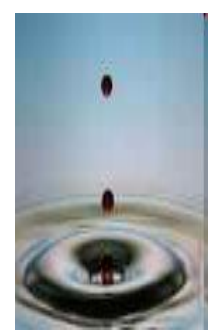




\section{Alia Hanafi}

pluralis fractus (جمع تكسير) which alters singular to plural by adding alif and سرادقات ד سرادق (awnings, tents) and بيمارستانات ج بيمارستان. (Hospitals) It is to be noticed also the exchanging of the letter $\boldsymbol{\zeta}$ to $\boldsymbol{\omega}$ which terminates the substantive since they are of the same group (the sibilants' letters $j$ (ص، س،).

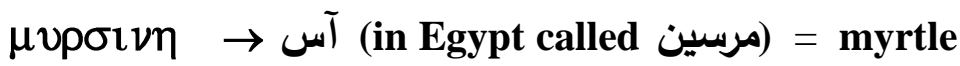

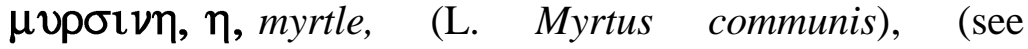
Inscriptiones Graecae $1^{2} .313 .150,2^{2} .949 .18,1235.14$, Theophrastus Philosophus (iv/iii BC.) HP1.14.4, etc. Archilochus Lyricus (vii BC.) 29, Lysippus Comicus (v BC.) 9, Alexander Rhetor (ii AD.) 98.25, Aristoteles Philosophus

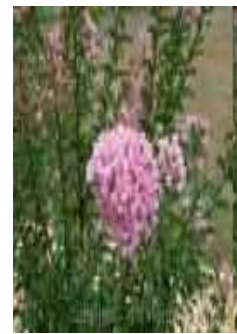
(iv BC.) $H A 627^{\mathrm{b}} 18$, and $\mu v \rho \sigma i v i v o s, \eta$, ov $=\mu v p p \imath v o \zeta$, of myrtle, Dsc.1.39, and $\mu v \rho \sigma \nu \omega \nu, \omega \nu \circ \zeta, 0$, myrtle-grove, Id.Ra.156, Aesopus Fabularum Scriptor 194, Philostratus Sophista (ii/iii AD.) Im.2.1.

It may be noticed also the similarities in Arabic to Greek words: on meeting $\kappa \rho \alpha \mu \beta \eta$, you may notice that the Arabic for "cabbage" kromb ${ }^{\mathbf{1 8}}$. Taking Avicenna"s second book "Simple medicament" as an example, and by analysis of the botanic names presented in this book showed that literal translation was found in 14 words (4.964539\%), Arabization was found in 28 words $(9.929078 \%)$, transliteration was found in 37 words $(13.12057 \%)$ and translation was found in 205 words $(72.69504 \%)$. The following table and figures summarizes

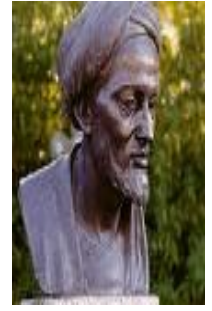
these results.

Table 1: Methods of transfer of medical terms

\begin{tabular}{|l||c||c||}
\hline \hline Methods of transfer & Number of words & Percent \\
\hline \hline Literal translation & 14 & $4.964539 \%$ \\
Arabization & 28 & $9.929078 \%$ \\
Transliteration & 37 & $13.12057 \%$ \\
Translation & 205 & $72.69504 \%$ \\
\hline
\end{tabular}


The interaction between Greek and Arabic Medical Terms

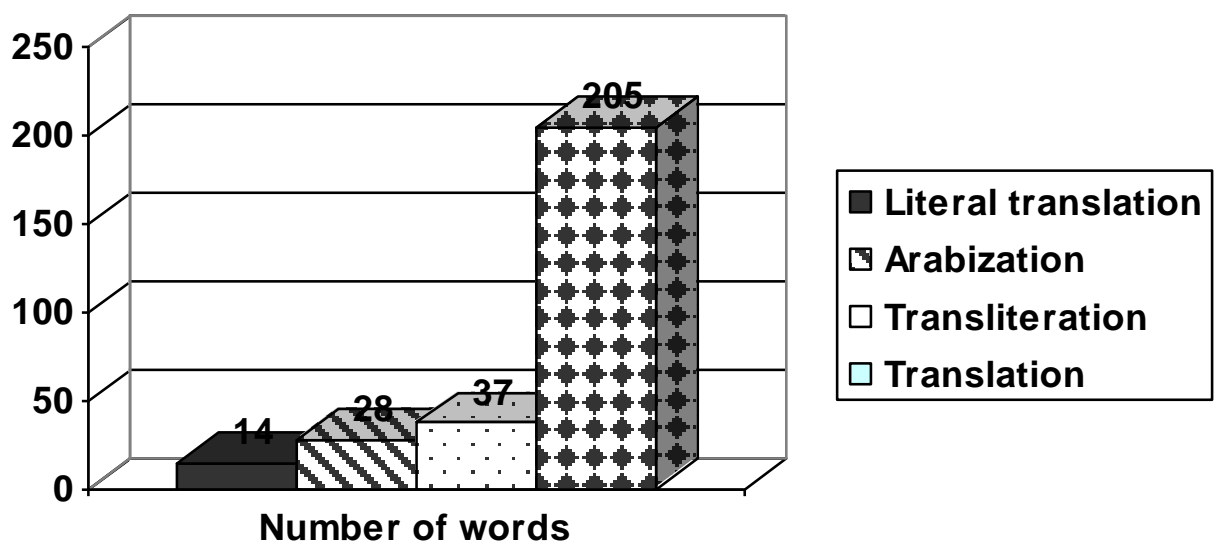

Fig 1: Methods of transfer of medical terms by number of words

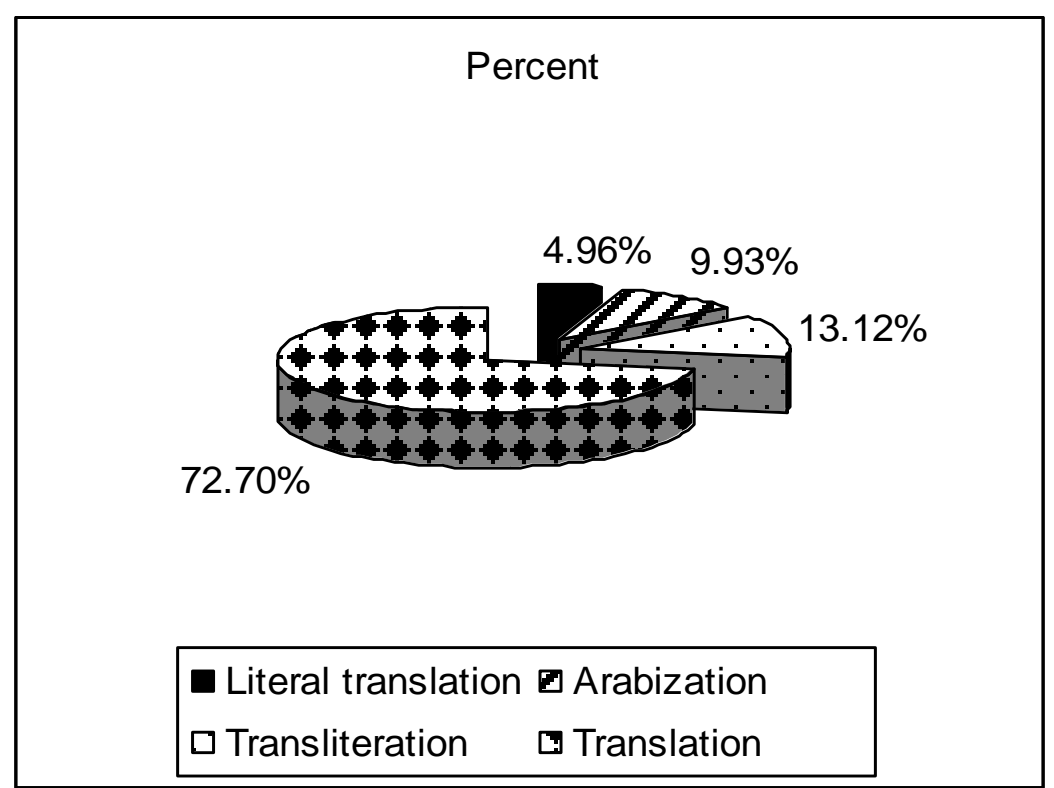

Fig 2: Methods of transfer of medical terms by percent V. 


\section{Alia Hanafi}

Full analysis of the only Botanic Greek ${ }^{19}$ terms in Ibn Sina' AlCanon fi Al-ar Oib ${ }^{20}$, the second book, the second tractate is shown in the following tables:

\begin{tabular}{|c|c|c|c|c|c|}
\hline \multicolumn{2}{|c|}{$\begin{array}{c}\text { Literal translation } \\
14 \text { words }\end{array}$} & \multicolumn{4}{|c|}{$\begin{array}{c}\text { Arabization } \\
28 \text { words }\end{array}$} \\
\hline$\mu v o s \omega \tau \alpha$ & آذان الفار & $\mu v \rho \sigma i v \eta$ & آس آس (in & $\gamma \varepsilon \nu \tau i \alpha \nu \eta$ & جنطيانا \\
\hline$\alpha \sigma \tau \eta \rho$ A & أسطاروس & $\sigma \kappa \imath \lambda \lambda \alpha$ & سقلا & $\alpha \mu \omega \mu \circ v$ & حماما \\
\hline 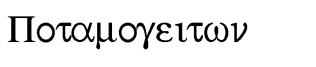 & جار النهز & $\alpha \gamma \alpha \lambda \circ x \circ v$ & أغالوجى & 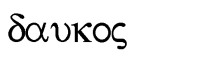 & 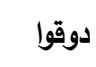 \\
\hline 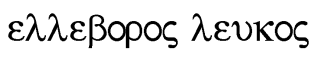 & خربق أبيض & $\varepsilon \pi \imath \theta u \mu \circ \nu$ & افيثمون & $\zeta \mathrm{\imath} \gamma \gamma \mathrm{\imath} \beta \varepsilon \rho \mathrm{\imath}$ & زنجبيل \\
\hline$\varepsilon \lambda \lambda \varepsilon \beta o p \circ \varsigma \mu \varepsilon \lambda \alpha \varsigma$ & خربق أسود & $\alpha \psi i v \theta i o v$ & افسنتين & $\sigma \alpha \gamma \alpha \pi \eta \nu \circ \nu$ & ج ج السبيذ \\
\hline$\pi \varepsilon \nu \tau \varepsilon \phi \nu \lambda \lambda \circ \nu$ & خمسة أورلق & $\alpha \kappa \alpha \nu \theta 10 v$ & أقسون & $\sigma 1 \lambda \lambda \nu \beta \circ \nu$ & سلوثن \\
\hline $1 \pi \pi$ ovpls & ذنب الخيل & $\alpha \kappa \alpha \nu \theta \imath \mathrm{\nu}$ & أقسون & $\sigma \eta \sigma \alpha \mu \circ \nu$ & سمسم \\
\hline$\tau \rho \imath \chi о \mu \alpha \nu \varepsilon \varsigma$ & شعر الغول & ipis & 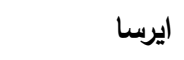 & $\kappa \alpha \nu \nu \alpha \beta i \varsigma$ & قتب \\
\hline 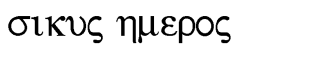 & قثاء بستانى & $\pi \varepsilon \pi \lambda \circ \varsigma$ & 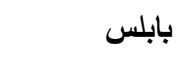 & $\kappa \alpha \pi \pi \alpha p i \varsigma$ & كبر \\
\hline olkvs $\alpha \gamma p i o s$ & قاء برى قاء & $\pi \alpha \pi v p o \varsigma$ & بردى & $\kappa \alpha \rho \omega$ & كراويا \\
\hline$\kappa \rho \alpha \mu \beta \eta \alpha \gamma \rho 1 \alpha$ & كرنب برى & $\beta \alpha \lambda \sigma \alpha \mu \circ \nu$ & 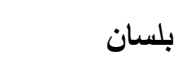 & конtvov & كمون \\
\hline 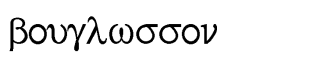 & ل ل ل ل & $\pi \circ \nu \tau \iota \kappa \alpha$ & بندق & $\mu \eta о \nu$ & مو \\
\hline$\alpha \rho v o \gamma \lambda \omega \sigma \sigma o v$ & لسان الحمل & $\theta \varepsilon \rho \mu \circ \varsigma$ & بندق & 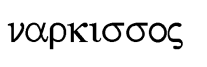 & 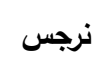 \\
\hline $\begin{array}{l}\alpha \mu v \gamma \delta \alpha \lambda \eta \gamma \lambda v \kappa \varepsilon 1 \\
\alpha\end{array}$ & لوز حلو & $\theta \alpha \psi 1 \alpha$ & تفسيا & $\rho \circ \delta \alpha$ & ورد \\
\hline
\end{tabular}

V) 
The interaction between Greek and Arabic Medical Terms

\begin{tabular}{|c|c|c|c|}
\hline \multicolumn{4}{|c|}{$\begin{array}{c}\text { Transliteration } \\
37 \text { words } \\
\end{array}$} \\
\hline$\varepsilon \beta \varepsilon v O \zeta$ & أبنوس & $\sigma 1 \sigma \alpha \rho o v$ & سيسارون \\
\hline$\alpha \sigma \alpha \rho o v$ & أسارون & $\sigma \varepsilon \sigma \varepsilon \lambda 1 \varsigma$ & سيساليوس \\
\hline$\sigma \tau 01 \chi \alpha \varsigma$ & الأسطوخوذوس & olov & سيون \\
\hline$\imath \tau \varepsilon \alpha$ & أطا & $\tau \rho i \phi v \lambda \lambda \circ \nu$ & طريفلن \\
\hline$\alpha \kappa \alpha \kappa \imath \alpha$ & أقاقيا & 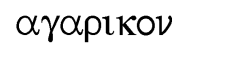 & غاريقون \\
\hline$\alpha \lambda v \sigma \sigma o v$ & ألوسن & $\gamma \alpha \lambda 10 \nu$ & غاليون \\
\hline$\alpha v \alpha \gamma \alpha \lambda \lambda 1 s$ & أناغالس & $\pi p \alpha \sigma 10 \nu$ & فراسيون \\
\hline$\eta \delta v \sigma \alpha \rho \circ v$ & ايدوصارون & $\pi \imath \sigma \tau \alpha \kappa \imath \alpha$ & فستق \\
\hline$\alpha \nu \eta \sigma o \nu$ & انيسون & $\pi \varepsilon \tau \rho \circ \sigma \varepsilon \lambda \iota \nu \circ \nu$ & فطراساليون \\
\hline$\beta \rho \varepsilon \tau \tau \alpha \nu \kappa \eta$ & برطانيقى & $\phi o v$ & فو \\
\hline Bo $\lambda \beta O \varsigma$ & بلبوس & $\kappa \alpha \rho \delta \alpha \mu \omega \mu \circ \nu$ & قردمانا \\
\hline$\chi \varepsilon \lambda{ }_{1} \delta$ oviov & خاليدونيون & 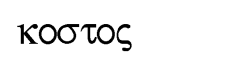 & قسط \\
\hline$\chi \alpha \mu \alpha \imath \lambda \varepsilon \omega \nu$ & خامالاون & $\kappa 1 \sigma \sigma \circ \varsigma$ & فسوس \\
\hline 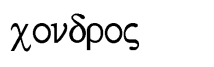 & خندروس & 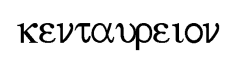 & قنطوريون \\
\hline$\delta \rho v o \pi \tau \varepsilon \rho ı \varsigma$ & دروبطارس & $\chi \alpha \mu \alpha 1 \delta \rho v \varsigma$ & كمادريوس \\
\hline$\sigma \phi O \nu \delta v \lambda 10 v$ & سفندوليون & $\chi \alpha \mu \alpha \imath \pi \imath \tau v \varsigma$ & كمافيطوس \\
\hline$\sigma \kappa \alpha \mu \mu \omega \nu l \alpha$ & سقونيا & $\lambda \circ \beta 1 \alpha$ & لوبيا \\
\hline$\sigma \kappa o p \delta i o v$ & سقورديون & $\lambda \varepsilon 1 \mu \omega \nu 10 \nu$ & ليمونيون \\
\hline$\sigma \nu \mu \phi \nu \tau O \nu$ & سومفوطون & & \\
\hline
\end{tabular}

\begin{tabular}{|c|c|c|c|c|c|}
\hline \multicolumn{6}{|c|}{ Translation 205 words } \\
\hline$\beta \rho \alpha \theta v$ & أبهل & $\varepsilon v \zeta \omega \mu \mathrm{ov}$ & جرجير & $\pi \varepsilon \rho 1 \sigma \tau \varepsilon p 10 \nu$ & رعى الحمام \\
\hline$\phi v \tau o v$ & أترج & $\sigma \tau \alpha \phi v \lambda i v \circ \varsigma$ & جزر ار & $\varepsilon \lambda \alpha \phi \circ \beta ం \sigma \kappa \circ \nu$ & رعى الأبل \\
\hline$\kappa о \kappa \kappa v \mu \eta \lambda \varepsilon \alpha$ & آجاص & $\pi 0 \lambda 10 v$ & جعدة & $\rho \circ \alpha$ & رمان \\
\hline oxolvos & أنخر & $\sigma \eta \sigma \alpha \mu \circ \varepsilon 1 \delta \varepsilon \varsigma$ & جلبهنك & $\alpha \rho 1 \sigma \tau 0 \lambda \circ \chi \varepsilon 1 \alpha$ & 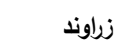 \\
\hline Bpvov & أشنة & $\beta \alpha \lambda \alpha \nu \sigma \tau 10 \nu$ & جلنار & $\mu \varepsilon \sigma \pi \mathrm{\imath} \lambda \circ \nu$ & زعرور \\
\hline$\pi \alpha \rho \theta \varepsilon v i o v$ & أقحوان & $\sigma v \kappa \alpha \mu о \rho о \nu$ & جميز & крокољ & زعفران \\
\hline$\mu \varepsilon \lambda 1 \lambda \omega \tau \tau^{\prime}$ & أكليل & $\kappa \alpha \rho \cup \alpha \beta \alpha \sigma \iota \kappa \iota \kappa \alpha$ & جوز & $\alpha \lambda 1 \sigma \mu \alpha$ & زمارة الراعى \\
\hline$\pi \alpha \lambda 10$ vo & أم غيلان & $\theta v \mu \circ s$ & حاشا & $\alpha \nu \theta v \lambda \lambda 1 \varsigma$ & الزهره \\
\hline
\end{tabular}




\section{Alia Hanafi}

\begin{tabular}{|c|c|c|}
\hline 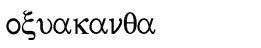 & أنبرباريس & $\delta \alpha \phi v i \delta \varepsilon \varsigma$ \\
\hline$\sigma 1 \lambda \phi 1 \circ \nu$ & انجدان & $\tau \varepsilon \rho \mu \imath \nu \theta \circ \varsigma$ \\
\hline$\alpha \kappa \alpha \lambda \eta \phi \eta$ & أنجرة & $\sigma \kappa o \lambda v \mu \circ \varsigma$ \\
\hline$\alpha \nu \theta \varepsilon \mu 1 \varsigma$ & بابونج & $\kappa \alpha \rho \delta \alpha \mu \circ \nu$ \\
\hline$\alpha \kappa \alpha \nu \theta \alpha \lambda \varepsilon v \kappa \eta$ & باذاورد & $\pi \eta \gamma \alpha \nu \circ v \alpha \gamma p i o v$ \\
\hline$\mu \varepsilon \lambda 1 \sigma \sigma \circ \phi \nu \lambda \lambda{ }^{\prime}$ & بادرنجويه & $\tau \rho 1 \beta 0 \lambda \circ \varsigma$ \\
\hline$\omega \kappa \iota \mu о \nu$ & باذرج & $\varepsilon \lambda \xi ı \nu \eta$ \\
\hline$\kappa v \alpha \mu \circ \varsigma \mathrm{E \lambda \lambda \eta \nu 1 \kappa \circ \varsigma}$ & بقلا & $\lambda$ ขКiov \\
\hline$\beta \alpha \lambda \alpha \nu \circ \varsigma$ & بان بان & $\tau \eta \lambda i s$ \\
\hline$\alpha \delta 1 \alpha v \tau o v$ & برسياوشان & $\lambda \alpha \pi \alpha \theta \circ v$ \\
\hline$\alpha \rho \tau \varepsilon \mu 1 \sigma \imath \alpha$ & برنجاسف & $\varepsilon \rho \varepsilon \beta 1 \nu \theta \circ \varsigma$ \\
\hline$\psi v \lambda \lambda \lambda 10 \nu$ & بزرقطونا & китрољ \\
\hline$\lambda \iota \nu \circ \sigma \pi \varepsilon \rho \mu \circ \nu$ & بزر كتان & $\lambda \omega \tau{ }^{\prime}$ \\
\hline$\mu \alpha \kappa i p$ & بسباسه & $\pi v p o 1$ \\
\hline коvр $\alpha \lambda 10 \mathrm{v}$ & بسد & 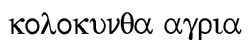 \\
\hline$\pi 0 \lambda v \pi \circ \delta 10 \nu$ & بسفايج & $\lambda \varepsilon v \kappa \eta$ \\
\hline кронvоv & بصل & $\alpha i \gamma \varepsilon i p o s$ \\
\hline$\pi \circ \lambda v \gamma \circ v \circ v$ & بطباط & $\mu о \lambda \circ \chi \eta$ \\
\hline$\tau \varepsilon \rho \mu \imath \nu \theta \circ \varsigma$ & بطم & $\sigma \iota \nu \eta \pi \mathrm{i}$ \\
\hline$\pi \varepsilon \pi \omega \nu$ & بطيخ & $\kappa \varepsilon p \alpha \tau 1 \alpha$ \\
\hline$\alpha \nu \delta \rho \alpha \chi \nu \eta$ & بقلة حمقاء & $\theta p 1 \delta \alpha \xi$ \\
\hline$\beta \lambda 1 \tau \circ \nu$ & بقلة يمانية & $\mu \eta \kappa \omega \nu$ \\
\hline Spvs & بلوط & $\sigma \alpha \tau u p i o v$ \\
\hline 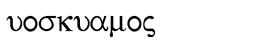 & بنج & opxis \\
\hline$\alpha \gamma \nu o s$ & بنجنكثت & $\alpha \lambda \theta \alpha 1 \alpha$ \\
\hline ov & بنفسج & $\alpha \sigma \phi \circ \delta \varepsilon \lambda \circ \varsigma$ \\
\hline$\alpha \phi \alpha \kappa \eta$ & بيقية & Пврбıк $\alpha \mu \eta \lambda \alpha$ \\
\hline$v \alpha \sigma \kappa \alpha \phi \theta 0 v$ & بنك & $\alpha \sigma \pi \alpha \lambda \alpha \theta \circ \varsigma$ \\
\hline$\beta o v \phi \theta \alpha \lambda \mu \circ v$ & بنك & $\kappa \iota \nu \alpha \mu \omega \mu \mathrm{ov}$ \\
\hline$\phi \lambda о \mu \circ \varsigma$ & بوصير & $1 \xi \circ \varsigma$ \\
\hline$\mu \eta \lambda \varepsilon \alpha \varsigma$ & بوصير & $\pi \tau \varepsilon \lambda \varepsilon \alpha \varsigma$ \\
\hline$\mu о \rho \varepsilon \alpha$ & توت & vmpiov \\
\hline$\varepsilon \rho \nu \sigma \iota \mu \circ \nu$ & تودرى & $\pi \tau \alpha \tau \alpha \nu \circ \varsigma$ \\
\hline$\sigma \nu \kappa \alpha$ & تين & $\alpha \imath \gamma \imath \lambda \omega \psi$ \\
\hline 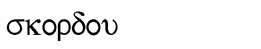 & ثوم & $\mu \alpha \rho \alpha \theta \circ \nu$ \\
\hline$\alpha \gamma \rho \omega \sigma \tau \iota \varsigma$ & ثيل & $\alpha \lambda \varepsilon v i o v$ \\
\hline 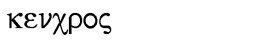 & جاروس & $\rho \alpha$ \\
\hline$\pi \alpha v \alpha \kappa \varepsilon \varsigma$ & جاوشير & $\mu \eta \delta 1 \kappa \eta$ \\
\hline
\end{tabular}

\begin{tabular}{|c|c|}
\hline حب الغار الغ & $v \sigma \sigma \omega \pi \circ \varsigma$ \\
\hline حبة الخضرا & $\pi \alpha \nu \alpha \kappa \varepsilon \varsigma$ A $\sigma \kappa \lambda \eta \pi l<\varepsilon l>O \nu$ \\
\hline حرشف - l & $\alpha \gamma \rho 1 \varepsilon \lambda \alpha 1 \alpha$ \\
\hline 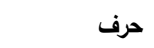 & $\mu \alpha \lambda \alpha \beta \alpha \theta-\rho \circ v$ \\
\hline 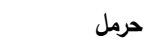 & $\pi \eta \gamma \propto \nu \circ \nu$ \\
\hline 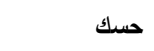 & $\lambda v \chi \nu i \varsigma \sigma \tau \varepsilon \phi \alpha \nu \omega \mu \alpha \tau \imath \kappa \eta$ \\
\hline حشيشة الزجاج & $\pi \tau \varepsilon \rho ı \varsigma$ \\
\hline 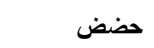 & $\alpha \nu \delta \rho \alpha \phi \alpha \xi v \varsigma$ \\
\hline حلبه & $\kappa v \pi \alpha \rho i \sigma \sigma o \varsigma$ \\
\hline جماض & $\beta \eta \chi 10 \nu$ \\
\hline حمص & $\theta v \mu \beta \rho \alpha$ \\
\hline 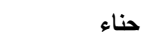 & $\kappa v \pi \varepsilon \rho \circ \varsigma$ \\
\hline حندقوقى & $\kappa v \delta \omega \nu 1 \alpha$ \\
\hline حنطه & $\alpha \sigma \pi \lambda \eta \nu \circ s$ \\
\hline حنظل ال & $\sigma \varepsilon v \tau \lambda \circ \nu$ \\
\hline حور - ت حور & $\kappa \alpha \sigma \sigma 1 \alpha \varsigma$ \\
\hline حور رومى & pous \\
\hline 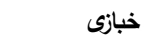 & $\sigma \mu v p v i o v$ \\
\hline 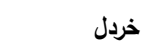 & 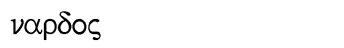 \\
\hline خرنوب & $\kappa \circ \lambda \chi 1 \kappa \circ \nu$ \\
\hline 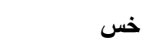 & кpivov \\
\hline 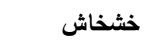 & $\kappa \alpha \pi \nu \circ \varsigma$ \\
\hline خصى الثعلب & $\alpha \nu \eta \theta 0 \nu$ \\
\hline خصى الكلب & $\pi \mathrm{\imath} \tau \circ v \sigma \alpha$ \\
\hline 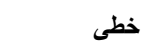 & $\kappa \varepsilon \delta \rho \circ \varsigma$ \\
\hline 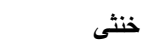 & $\alpha \lambda \phi i \tau \circ \nu$ \\
\hline خوخ & $\alpha \nu \varepsilon \mu \omega \nu \eta$ \\
\hline دار شيشعان & $\alpha \kappa \tau \eta$ \\
\hline دار صينى & $\gamma \circ \gamma \gamma \cup \lambda \eta$ \\
\hline دبق ابق & $\alpha \gamma \chi \circ v \sigma \alpha$ \\
\hline 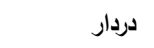 & $\mu \varepsilon \lambda \alpha \nu \theta i o \nu$ \\
\hline 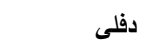 & бepiфov \\
\hline 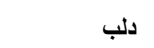 & $\lambda \varepsilon \pi 1 \delta 10 \nu$ \\
\hline 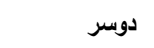 & $\alpha 1 p \alpha$ \\
\hline 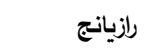 & $\alpha \lambda v \eta$ \\
\hline 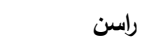 & $\sigma \tau \rho \circ \beta \imath \lambda \circ 1$ \\
\hline 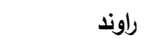 & $\phi \alpha \kappa \circ \varsigma$ \\
\hline رطبه & $\mu v \rho ı \kappa \eta$ \\
\hline
\end{tabular}

روفا يابس

زوفرا

ريتون 
- The interaction between Greek and Arabic Medical Terms

\begin{tabular}{|c|c|c|c|c|c|}
\hline \multicolumn{6}{|c|}{$\begin{array}{c}\text { Translation (Cont.) } \\
205 \text { words }\end{array}$} \\
\hline$\sigma \tau \rho \circ \beta 1 \lambda \circ \mathrm{ol}$ & صنوبر & 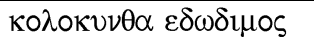 & قرع & $\lambda \alpha \theta u p i s$ & ماهوبدانة \\
\hline$\phi \alpha \kappa \circ \varsigma$ & 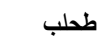 & $\kappa \alpha \lambda \alpha . \mu \omega \nu$ & قصب الذريرة & $\phi 1 \lambda \cup p \alpha$ & 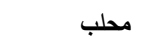 \\
\hline$\mu v p ı \kappa \eta$ & طرفاء & $\kappa \alpha \lambda \alpha . \mu \circ \varsigma \alpha \rho \omega \mu \alpha \tau \imath \kappa \circ \varsigma$ & قصب & $\mu \varepsilon \lambda 1 \alpha$ & 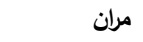 \\
\hline$\pi v \rho \varepsilon \theta \rho o s$ & عاقر قرحا & $\alpha \sigma \kappa \lambda \eta \pi 1 \alpha \varsigma$ & 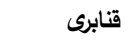 & $\sigma \alpha \mu \psi \circ \chi^{\prime} \propto \nu$ & 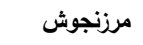 \\
\hline$\phi \alpha \kappa о \varsigma$ & 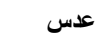 & $\alpha \beta p o \tau o v o v$ & ق قيصوم & $\mu \varepsilon \lambda 1 \sigma \sigma \circ \phi \nu \lambda \lambda \circ \nu$ & 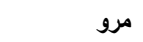 \\
\hline$\lambda \varepsilon \circ \nu \tau \circ \pi \varepsilon \tau \alpha \lambda \circ \nu$ & عرطنيثا & $\lambda \iota \gamma v \sigma \tau \iota \kappa о \nu$ & كاشم & $\delta 1 \kappa \tau \alpha \mu \nu \circ \nu$ & مشك طرامشير \\
\hline 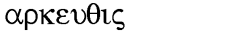 & عزعر & $\sigma \tau p v \chi v \circ v$ & كاكنج & 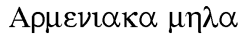 & شمش \\
\hline \multirow[t]{2}{*}{$\chi \varepsilon \lambda 1 \delta$ oviov $\mu \varepsilon \gamma \alpha$} & عروق & $\beta \alpha \tau \rho \alpha \chi 10 v$ & كبيكنج & $\sigma \chi 1 v o s$ & مصطكى \\
\hline & الصباغين & & & & \\
\hline$\kappa \eta \kappa i \varsigma$ & عفص & $\tau \rho \alpha \gamma \alpha \kappa \alpha \nu \theta \alpha$ & 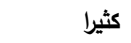 & $\sigma \tau \alpha \phi i \varsigma \alpha \gamma \rho 1 \alpha$ & 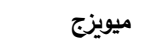 \\
\hline$\beta \alpha \tau \circ \varsigma$ & عليق & 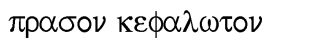 & 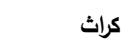 & $\alpha \mu \imath$ & ن انخواه \\
\hline$\sigma \tau \alpha \phi \nu \lambda \eta$ & عنب & opoßos & كرسنة & $\phi 01 v 1 \xi$ & 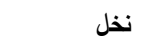 \\
\hline \multirow[t]{2}{*}{$\sigma \tau \rho \nu \chi v o v$} & عنب & $\sigma \varepsilon \lambda 1 v O \nu$ & 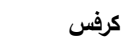 & $\eta \delta v \circ \sigma \mu \circ v$ & 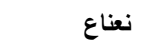 \\
\hline & الثعلب & & & & \\
\hline 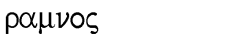 & عوسج & $\alpha \mu \pi \varepsilon \lambda \circ \varsigma$ olvoфopos & 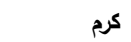 & $\varepsilon \rho \pi v \lambda \lambda \circ \varsigma$ & 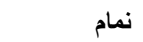 \\
\hline$\delta \alpha \phi \nu \eta$ & غار & kopiov & 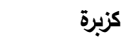 & вроно & هرطمان \\
\hline$\varepsilon v \pi \alpha \tau o p 10 \varsigma$ & 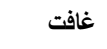 & $\alpha \kappa \alpha \kappa \alpha \lambda \lambda i \varsigma$ & كزمازك & $v v \mu \phi \alpha 1 \alpha$ & 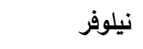 \\
\hline$\imath \tau \varepsilon \alpha$ & غرب & $v \delta v o v$ & كمأة & бepls & هندبا \\
\hline$\alpha \mu \pi \varepsilon \lambda \circ \varsigma \lambda \varepsilon \cup \kappa \eta$ & فاشرا & $\alpha \pi i o s$ & كثرى - ك & $\alpha \sigma \pi \alpha \rho \alpha \gamma \circ \varsigma$ & هليون \\
\hline$\alpha \mu \pi \varepsilon \lambda \circ \varsigma \mu \varepsilon \lambda \alpha \mathrm{l} v \alpha$ & فاشرستين & $\sigma \tau \rho \circ v \theta 1 o v$ & 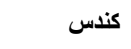 & $\alpha$ короv & وج \\
\hline$\gamma \lambda v \kappa v \sigma i \delta \eta$ & فاوانيا & $\kappa 1 \sigma \theta \circ \varsigma$ & 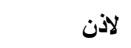 & $1 \sigma \tau \alpha \tau \iota \varsigma$ & وسمد \\
\hline$\rho \alpha \phi \alpha \nu i s$ & فجل & $\pi \varepsilon \rho \sigma \alpha 1 \alpha$ & اللبخ & $\mu \alpha \nu \alpha \delta \rho \alpha \gamma o p \alpha \varsigma$ & يبروح | يبرح \\
\hline$\pi \varepsilon \pi \varepsilon \rho \imath$ & فلفل & $\varepsilon \lambda \xi ı \nu \eta$ & 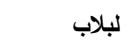 & $\tau 1 \theta v \mu \alpha \lambda \lambda \circ \nu$ & يتوع \\
\hline$\alpha \kappa ı s$ & فلنجمثك & $\alpha \rho o v$ & لوف ل ل ل & $\alpha$ короv & ينبوت \\
\hline$\kappa \alpha \lambda \alpha \mu \imath \nu \theta \eta$ & فوذنج & $\chi \alpha \mu \varepsilon \lambda \alpha 1 \alpha$ & مازريون & & \\
\hline \multirow[t]{2}{*}{$\varepsilon \rho v \theta \rho \circ \delta \alpha \nu \circ \nu$} & فوة & $\gamma \lambda \alpha v \kappa 10 \nu$ & ماميثا & & \\
\hline & الصباغين & & & & \\
\hline
\end{tabular}




\section{Alia Hanafi}

\section{Notas}

${ }^{1}$ The Cambridge History of Science: Eighteenth-century science / edited by Roy Porter, et al. Published by Cambridge University Press, 2003, Page 670.

${ }^{2}$ Rahman, A. 1996. A Perspective of Indian Science of Tenth-Eighteenth Centuries. In Science Philosophy and Culture-Multi Disciplinary Explorations (Part I) Edited by D.P. Chattopadhayaya and Ravinder Kumar. New Delhi: PHISPC. Pp. 396-426.

${ }^{3}$ Charles S. F. Burnett, Danielle Jacquart (eds.), Constantine the African and $\odot A l i \overline{I b n ~ A l-~}$ $\odot$ Abbās Al-Magūsī: The Pantegni and Related Texts. Leiden: Brill, 1995.

${ }^{4}$ Malaria was a common and endemic disease in the Classical era (see Sallares R. Malaria and Rome, Oxford, University Press, 2002, 1-123). Asclepiades (2nd century BC), while confirming the high incidence of periodic fevers in Rome, stressed the seriousness of quotidian fevers which often caused serious brain disease. (see Retief FP, Cilliers L. Malaria in Graeco-Roman times. Acta Classica 2004; 67: 127-137.). In the 1 st century the encyclopaedist, Celsus, clearly described three kinds of periodic fevers which almost certainly were malaria, viz. quotidian, tertian and quartan fevers. In an extraordinary observation he mentioned that tertian fevers would be mild when days between fever peaks were a pyrexia (benign tertian malaria), but more serious when fever never completely abated - malignant tertian malaria according to the classical description given above, and called hemitritaion (semi-tertian fever) by himself. Pliny the Elder (Pliny the Elder, Natural History, 28. 23, 83; 29.17.63; 30.98) summarized existing therapeutic regimens, including herbal remedies and magical procedures(see Francois Retief, Louise Cilliers, Pyrexia and Malaria in Antiquity, 2006, Vol. 96, No. 8 SAMJ p. 687 ff.).

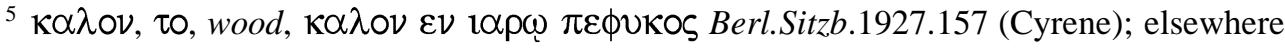
only in pl. $\kappa \alpha \lambda \alpha=\xi \nu \lambda \alpha, \operatorname{logs}$, for burning, $\kappa \alpha \gamma \kappa \alpha \nu \alpha \kappa$. h.Merc.112;

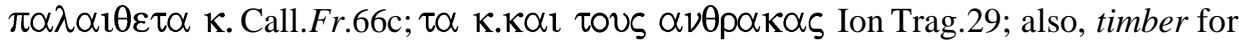
joiner's work, $\varepsilon \pi \imath \kappa \alpha \mu \pi \nu \lambda \alpha \kappa$. Hes.Op.427; esp. of ships, $\pi$ o $\tau \alpha \kappa \alpha \lambda \alpha(\kappa \alpha \lambda \alpha$ cod.) Ar.Lys.1253; $\varepsilon p \rho \varepsilon \imath \tau \alpha \kappa \alpha \lambda \alpha$ the ships are lost ( $\kappa \alpha \lambda \alpha$ codd.), X.HG1.1.23, Plu.Alc.28. ( $\kappa \alpha \lambda$ ov and $\kappa \eta \lambda$ ov (q.v.) perh. fr. ).

${ }^{6}$ Suidas Lexicographus (x AD.) vs. $\kappa \alpha \lambda \circ \pi \delta \alpha p 1 \alpha 1$.

${ }^{7}$ Edictum Diocletiani, ed. T. Mommsen \& H. Blümner, Der Maximaltarif des Diocletian, Berlin 1893; suppl. CIL iii pp. 1926 ff., 2208 ff, $2328^{57}$ ff.: cited where possible by Mommsen's chaps. \& lines, recently found portions by place of discovery Diokletians Preisedikt. Texte und Kommentare, S. Lauffer, Berlin 1971.; (Aeg. = Aegira; Clit. = Clitor; Delph. $=$ Delphi; Troez. $=$ Troezen); Geronthr. $=$ IG5(1)1115; Gyth. $=5(1) 1148$.

${ }^{8}$ Collins English Dictionary (1979), vs. pistachio.

${ }^{9}$ In ancient times, epilepsy was known as the "Sacred Disease" because people thought that epileptic seizures were a form of attack by demons, or that the vision experienced by persons with epilepsy were sent by the gods.(see Epilepsy, http://en.wikipedia.org/wiki/Epilepsy, and Harper, Douglas (2001). "epilepsy". Online Etymological Dictionary. Retrieved on 2005-06-05.). 


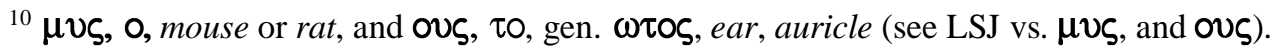

${ }^{11}$ Madwort is a low-growing Eurasian plant (Asperugo procumbens) having rough stems and small blue flowers. Alyssum, genus of low-growing, mostly perennial plants, also called madwort. Golden tuft, or rock madwort, and yellow tuft are spring-flowering.

12 Wright, W., A Grammar of the Arabic Language, Cambridge 1967, p. 7.

13 The philosopher's stone (L. lapis philosophorum; Gr. $\chi \rho v \sigma 0 \pi 01 \mathrm{\alpha}, \eta$ "the making of gold") is a legendary substance, supposedly capable of turning inexpensive metals into gold.; it was also sometimes believed to be an elixir of life, useful for rejuvenation and possibly for achieving immortality. In the 8th-century, Arab alchemist Jabir ibn Hayyan analyzed each classical element in terms of the four basic qualities of hotness, coldness, dryness, and moistness. Fire was both hot and dry, earth cold and dry, water cold and moist, and air hot and moist. He further theorized that every metal was a combination of these four principles, two of them interior and two exterior. From this premise, it was reasoned that the transmutation of one metal into another could be affected by the rearrangement of its basic qualities. This change would presumably be mediated by a substance, which came to be called al-iksir in Arabic. http://en.wikipedia.org/wiki/Philosopher\%27s_stone\#cite_note-3

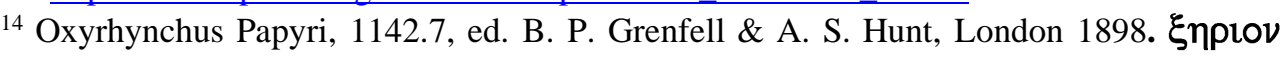
$(\delta \rho \alpha \chi \mu \omega \nu) 1 \delta$.

15 Alexander Trallianus Medicus [Alex.Trall.] (vi $\quad$ AD.) 1.15

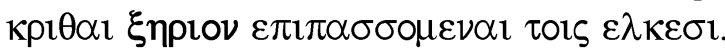

${ }^{16}$ Aethlius Historicus v B.C. Aët.6.65,

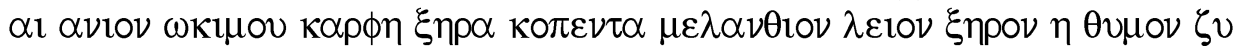

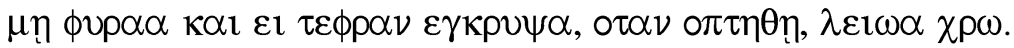

${ }^{17}$ Wright, W., op. cit. \& 26 p. 20.

${ }^{18}$ See W. G. Waddell, A Teacher of Classics in Egypt, The Classical Journal, Vol. 28, No. 7 (Apr., 1933), p 489.

${ }^{19}$ For Greek botanic terms see Pedanius Dioscorides Anazarbeus, De Matteria Medica, ed. Max Wellmann, Berolini 1907.

${ }^{20}$ For Arabic botanic terms see Avicenna, Al-Canon fi Al-@ Oib, Arabic version, ed. Boul9q 1284 AH./1877 AD. 